\title{
Mitochondrial DNA: A New Predictor of Diabetic Kidney Disease
}

\author{
Yajing Huang, ${ }^{1}$ Jingwei Chi, ${ }^{1}$ Fanxiang Wei, ${ }^{1}$ Yue Zhou, ${ }^{1}$ Yihai Cao, ${ }^{2}$ and Yangang Wang $\mathbb{D I D}^{1}$ \\ ${ }^{1}$ Department of Endocrinology, The Affiliated Hospital of Medical College of Qingdao University, Qingdao 266003, China \\ ${ }^{2}$ Department of Microbiology, Tumor and Cell Biology, Karolinska Institutet, Stockholm 171 77, Sweden
}

Correspondence should be addressed to Yangang Wang; wangyg1966@126.com

Received 21 April 2020; Accepted 25 June 2020; Published 15 July 2020

Academic Editor: Kazuhiro Shiizaki

Copyright $\odot 2020$ Yajing Huang et al. This is an open access article distributed under the Creative Commons Attribution License, which permits unrestricted use, distribution, and reproduction in any medium, provided the original work is properly cited.

Diabetic kidney disease (DKD) is a common cause of end-stage renal disease, and diagnosis and treatment in time can help delay its progress. At present, there are more and more studies on the pathogenesis of DKD; mitochondrial dysfunction plays an important role in DKD. The occurrence and development of DKD is closely related to epigenetic changes and the interaction between mtDNA, ROS, inflammatory factors, and endothelial damage, which continuously aggravates kidney. The change of mtDNA is both the cause of DKD and the result of DKD. It is of great significance to incorporate the change of mtDNA into the monitoring of patients with diabetes. Existing evidence indicates that changes in mtDNA copy number in blood and urine reflect mitochondrial dysfunction and the severity of DKD. However, large-scale, long-term follow-up clinical trials are still needed to determine the threshold range. By the time, mitochondrial-targeted antioxidants will become a new method for the treatment of DKD and other diabetic complications; mtDNA also can be a therapeutic target for them.

\section{Introduction}

Worldwide, the incidence of diabetes mellitus (DM) has increased rapidly and has affected more than 380 million people, of which China has the highest rate of diabetes patients, approximately 98.4 million [1]. Diabetic kidney disease (DKD) is the most serious microvascular disease in diabetes. In developed countries, DKD is the main cause of end-stage renal disease, accounting for about $44.5 \%$ of cases [2]. DKD is defined as a syndrome that is characterized by leakage of protein (in particular albumin), metabolites, and ions into the urine, changes in the glomerular filtration rate (GFR), and an increased risk of cardiovascular disease (CVD) and stroke [3]. The progressive exacerbation of DKD is manifested by the continued exacerbation of albuminuria and the gradual decline of glomerular filtration rate, which eventually leads to complete loss of renal function [4]. Before the emergence of clinical albuminuria (Mogensen stage III), there will be more insidious disease stages, during which the glomerular filtration rate is normal or highly filtered (Mogensen stages I and II), but there are no simple detection methods for diagnosis at present. Tight glucose control and antihypertensive drugs are now recognized as the main means of preventing early clinical DKD $[4,5]$. New antidiabetic drugs have renal protection while controlling blood glucose. For example, sodium glucose transporter 2 (SGLT2) inhibitors can reverse the hemodynamic changes observed in the afferent and efferent arteries and reduce glomerulosclerosis [6]. Angiotensin-converting enzyme inhibitors (ACEI) and angiotensin II receptor blockers (ARB) have become the first-line drugs for patient with DKD to control blood pressure and improve the prognosis of DKD. But these drugs can only delay the development of $\mathrm{DKD}$, but cannot reverse DKD [4].

The main histological features of the disease are glomerular basement membrane (GBM) thickening, glomerular mesangial expansion (GME), podocyte loss, glomerular sclerosis (GS), and progressive general fibrosis [7, 8]. Renal fibrosis is the ultimate pathological change of DKD. It is caused by a variety of mechanisms including renal hemodynamic changes, oxidative stress, inflammatory response, hypoxia, and activation of the renin-angiotensin-aldosterone system (RAAS) $[9,10]$. The kidney is a high metabolic organ; increasing evidence indicates that reactive oxygen species (ROS) overproduction is a common cause of kidney metabolic changes and hemodynamic changes [11]. 
Increased mitochondrial superoxide can lead to hyperglycemia, mitochondrial DNA (mtDNA) damage, and activation of apoptotic pathways [12]. Changes in mtDNA are closely related to $\operatorname{DKD}[3,13]$. In the prediabetes period, fasting blood glucose and $\mathrm{HbA} 1 \mathrm{C}$ had not changed significantly. But mtDNA NADH-6 (ND6) and D-loop had been methylated in these patients, and ND6 and D-loop methylation were closely related to insulin resistance [14]. Changes in mtDNA preceded DKD's bioenergy dysfunction and induced inflammatory response [15]. mtDNA changes were observed in tissue, blood, and urine. Mitochondrial protection has become a new target for the treatment of DKD [16]. We believe that monitoring changes in mtDNA can predict the occurrence of DKD, and it also can be a target for early intervention to delay the progress of $\mathrm{DKD}$.

\section{2. mtDNA Features}

Mitochondrion is a unique organelle that plays an important role in energy production, programmed cell death, calcium homeostasis, and synthesis of lipids, amino acids, and heme. Unlike the nuclear genome, mitochondria have their own genome, although the genome encodes only 13 peptides involved in oxidative phosphorylation [17]. However, these peptides are the basic components involved in the oxidative phosphorylation chain; they are essential for ATP production during respiration [17]. The double-stranded structure of mtDNA consists of a guanine-rich heavy $(H)$ strand and a light $(L)$ strand [15]. mtDNA is particularly vulnerable to damage, especially through the generation of oxidative lesions. First, mtDNA resides in close proximity to the site of ROS production in the mitochondrial membrane. Second, mtDNA replication proceeds via an asymmetric route, resulting in parts of the heavy strand existing as singlestranded structures for extended periods of time; this can lead to the spontaneous deamination of nucleotides [18]. Compared with genomic DNA, lower concentrations of ROS can cause damage to mtDNA, and under long-term oxidative stress, mtDNA damage is repaired more slowly than genomic DNA [19]. Impaired mtDNA and disrupted mitochondrial genome integrity play important roles in the development of severe early-onset and chronic aging-related diseases [19]. It is becoming increasingly clear that long-term, tiny mtDNA damage is not only related to the aging process, but may also be closely related to diabetes and its complications [20].

During early DKD, the ATP demand of kidney cells increased due to ultrafiltration, and increased ATP synthesis was caused by glycolysis. Chronic hyperglycemia disrupts the bioenergetic balance between the kidney's energy requirements and the supply of metabolic fuel, which is conducive to the production of ROS [21]. mtDNA in the blood can cross the kidney barrier and is excreted in the urine. Therefore, the detection of mtDNA in blood and urine can be used as a metabolic marker of early DKD (Table 1).

\section{Change of mtDNA in DKD}

3.1. Change of mtDNA in Tissues. Changes in mtDNA in tissues were found in pancreatic islet $\beta$ cells [26], skeletal muscle cells [27], animal models of diabetic retinopathy [28], and models of diabetic peripheral neuropathy [29] in diabetic patients. These include mitochondria morphological damage and decreased mtDNA copy number. Besides, oxidative mtDNA damage and mtDNA deletion were also observed in streptozotocin-induced diabetic rats [30]. In DKD mice, damage to mtDNA was localized to glomerular endothelial cells [12]. While in patients with DKD, mitochondrial fragmentation was specifically presented in tubules, but not in podocytes of DKD patients [25]. This contradicts the findings in animal models. The evolution of renal pathological changes in DKD mouse models is different from DKD patients [31]. When exploring the therapeutic targets of $\mathrm{DKD}$, it is important to choose the appropriate mouse model.

3.2. Change of $m t D N A$ in Blood. Sharma first reported that the copy number of mtDNA could be used as a new biomarker for DKD [13]. High glucose conditions downregulated the mtDNA content in mouse podocytes and promoted the release of mtDNA extracellularly. Due to the damage to mitochondrial biology, the amount of mtDNA in tissues (such as heart, liver, pancreas, and kidney) in the DM model decreased, while the amount of mtDNA in peripheral blood increased in patients with DM. Early increase in mtDNA copy number is closely related to insulin resistance and increased inflammatory factors $[22,24,32]$. Al-Kafaji et al. observed similar reductions in mtDNA copy number in peripheral blood in clinical trials. Reduced mtDNA copy number was negatively correlated with albuminuria and risk factors for DKD and positively correlated with estimated glomerular filtration rate [23]. In the early stage of DM, the mtDNA copy number in peripheral blood increased, and in patients with $\mathrm{DKD}$, the mtDNA copy number in peripheral blood decreased. This indicates that changes in mtDNA copy number in peripheral blood can predict the occurrence of DKD. Regular monitoring of mtDNA changes in peripheral blood in DM patients can be used as a predictor of DKD and reflect the severity of DKD.

3.3. Change of mtDNA in Urine. mtDNA in the blood can cross the kidney barrier and is excreted in the urine. Therefore, mtDNA in urine can also be used as a metabolic marker of DKD. Studies by Wei et al. showed that mtDNA levels in urine supernatants increased. It was associated with a decrease in mtDNA in the kidney $(r=-0.453, P=0.012)$ and was positively correlated with the severity of renal interstitial fibrosis $(r=0.300, P=0.005)$ [33]. In the DKD model, the essential mitochondrial genes of glomerular cells were significantly downregulated, and mitochondrial damage was increased. The damage marker 8-oxoguanine (8-oxodG) could be secreted into urine. In the early stage, mtDNA damage was limited to glomerular endothelial cells and accumulated over time, increasing urine secretion of 8 oxodG. The level of 8 -oxodG is related to the rate of progression of renal disease [12]. Cao et al. found that urine mtDNA/creatinine ratios increased in urine samples from patients with DM, especially those with DKD. At early 
TABLE 1: Clinical trial about mtDNA changes in DKD or T2DM.

\begin{tabular}{|c|c|c|c|c|c|c|}
\hline Author & Year & Samples $(n)$ & Treatment group & Control group & Observation index & $P$ value \\
\hline Sharma et al. [13] & 2013 & 32 & DKD & Healthy & Urinary mtDNA & $\leq 0.01$ \\
\hline Deng et al. [22] & 2019 & 244 & T2DM & Healthy & Plasma mtDNA & $<0.001$ \\
\hline Al-Kafaji et al. [23] & 2018 & 100 & $\mathrm{DKD}$ & T2DN & Plasma mtDNA & $<0.01$ \\
\hline Al-Kafaji et al. [23] & 2018 & 100 & DKD & Healthy & Plasma mtDNA & $<0.01$ \\
\hline Cao et al. [24] & 2018 & 77 & DKD & Healthy & Plasma mtDNA & 0.012 \\
\hline Cao et al. [24] & 2018 & 77 & DKD & Healthy & Urinary mtDNA/Cr ratio & $<0.001$ \\
\hline Jiang et al. [25] & 2019 & 108 & DKD & T2DM & Plasma mtDNA & 0.036 \\
\hline
\end{tabular}

microalbuminuria, urine changes occurred before changes in blood mtDNA [24]. Urine mtDNA changes occur earlier and are closely related to renal fibrosis, reflecting the progress of DKD.

Urine as an easily obtained and noninvasive sample source has an advantage over tissue and blood. Therefore, urine mtDNA is a good indicator for diagnosing DKD and is a prognostic indicator of DKD [34].

\section{Mitochondrial Oxidative Stress and mtDNA in DKD}

4.1. Mitochondrial Oxidative Stress in DKD. Kidney is the organ with the second highest oxygen consumption in our body; it is distinctly sensitive to mitochondrial dysfunction. Mitochondrial dysfunction contributes to the progression of DKD [35]. Mitochondrial dysfunction is characterized by the increase of mitochondrial ROS production, mitochondrial permeability transition (MPT) pore opening, and apoptosis [36]. Mitochondria are extremely vulnerable to hyperglycemia. Because mtDNA lacks protection and repair mechanisms, it can lead to a decrease in mtDNA [37]. The ATP content of the kidney in the DM mouse model decreased, and the copy number of mtDNA decreased, indicating that mitochondrial biological dysfunction and biogenesis dysfunction exist in the kidney [16]. The mechanisms of mitochondrial oxidative stress causing kidney damage include the following. (1) High glucose activates the JNK-CaMKII-Fis1 pathway, causing mitochondrial fragmentation, increased ROS, and JNK activation enhances podocyte apoptosis and renal tubular cell damage in mice [38]. (2) Mitochondrial dysfunction inhibits the AMPK-SIRT-1-PGC-1 $\alpha$ pathway and downregulates podocyte autophagy, and podocyte damage continues to accumulate, resulting in increased albuminuria $[16,39]$. (3) Excessive ROS induces the production of TGF- $\beta$, downregulates NO, and causes renal fibrosis and endothelial cell damage [40].

4.2. Mitochondrial Electron Transport Chain and mtDNA. ROS are produced during the normal operation of the mitochondrial electron transport chain (mtETC). When mtETC fails, ROS are overproduced. In kidney cells, hyperglycemia causes an increase in protein kinase C (PKC). The increase in PKC induces endothelial nitric oxide synthase (eNOS) production and increases the utilization of nitric oxide (NO) in the early stages of DKD [41]. Increased nitric oxide (NO) contributes to the activation of vascular endothelial growth factor (VEGF), which leads to endothelial dysfunction [42, 43]. When endothelial cell dysfunction occurs, endothelial cell mitochondrial function is inhibited and ROS production is increased. Excessive ROS inhibit mammalian target of rapamycin 1 (mTORC1) and AMP-activated protein kinase (AMPK) and affect the activation of peroxisome proliferator-activated receptor- $\gamma$ coactivator-1 $\alpha$ (PGC-1 $\alpha)$. As a major regulator of mitochondrial biogenesis, PGC- $1 \alpha$ is beneficial to mitochondrial biogenesis and the reparation of oxidative stress caused by ROS. The downregulation of PGC- $1 \alpha$ reduces the amount of mtDNA. Impaired gene expression leads to impaired synthesis of mtETC protein, accumulation of ROS, and continuous accumulation of damage to mtDNA, causing kidney damage [44, 45]. The DKD model also found that microtubular autophagic vacuoles and mitochondrial fragmentation were reduced under the microscope. The accumulation of these broken mitochondria also caused excessive production of ROS and aggravated oxidative stress [46].

4.3. Vicious Circle between ROS, mtDNA, and DKD. The mtDNA changes caused by mitochondrial oxidative stress aggravate the vicious cycle of DKD. The components are as follows: (1) high glucose leads to increased ATP demand, resulting in mtETC producing more ROS; (2) excess ROS destroys phospholipids, proteins, and nucleic acids; (3) ROS inhibits mitochondrial germinal growth by downregulating PGC- $1 \alpha$, and the amount of mtDNA decreases; (4) decreased synthesis of mtDNA-encoded subunits impairs the electron transport system and further augments the generation of superoxide promoting damage to mtDNA [47]; (5) ROS and damaged mtDNA continue to accumulate, which leads to podocyte and renal tubular cell damage, and aggravate by renal fibrosis.

\section{Inflammation and mtDNA in DKD}

mtDNA, when released extracellularly, could act as a damage-associated molecular pattern (DAMP) agent and cause inflammation [48]. Cao et al. pumped excess mtDNA into DM mice. Under diabetes, more mtDNA filtered by the kidney is associated with chronic sterile inflammation of the kidney [24]. The circulating cell-free mtDNA (ccf-mtDNA) can also activate the inflammasome (NLRP3). NLRP3 initiates inflammatory cascades that lead to activation of 


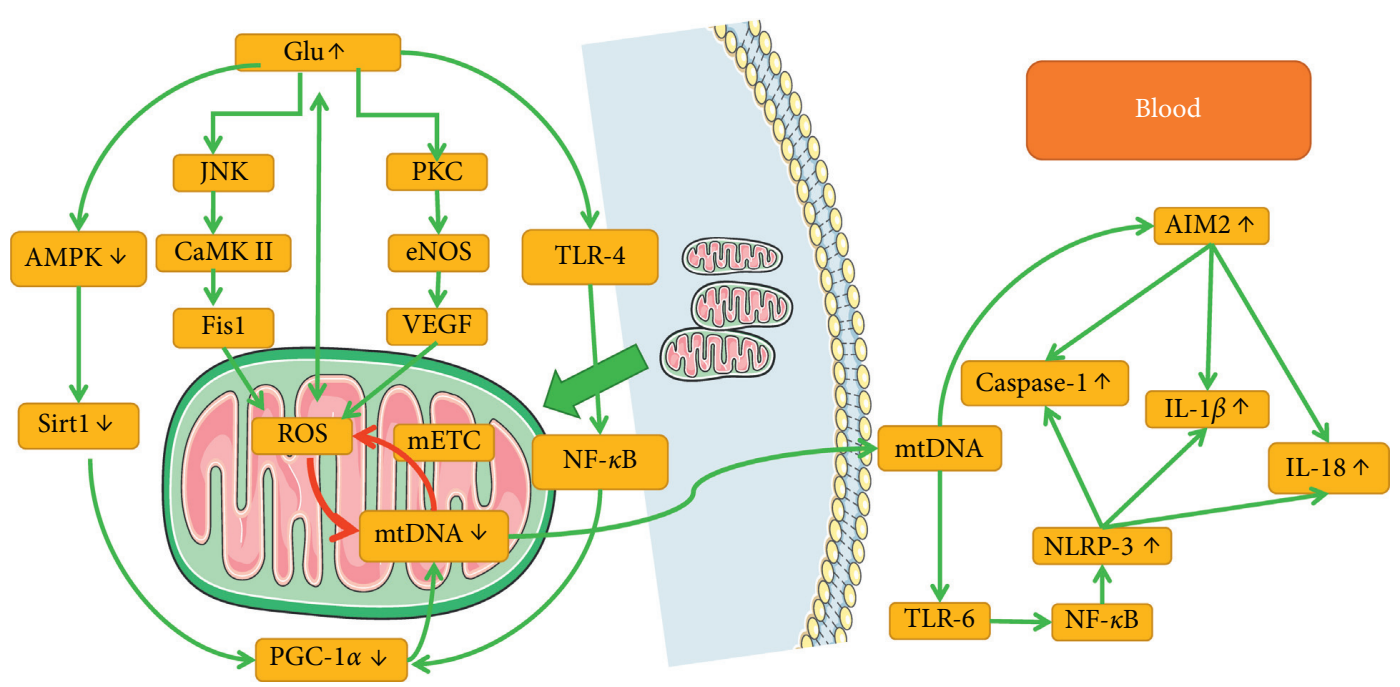

Figure 1: mtDNA, mitochondrial oxygen stress, and inflammation in DKD.

caspase- 1 and the production of IL- $1 \beta$ and IL- 18 through the TLR-NF- $\kappa$ B pathway $[49,50]$. The NLRP3 inflammasome links sensing of metabolic stress in the diabetic kidney disease [51]. ccf-mtDNA also acts as a critical signaling molecule in chronic inflammation via absent in melanoma 2 (AIM2) inflammasome activation. AIM2 also can promote caspase-1 activation and IL- $1 \beta$ and IL-18 secretion in macrophages [52]. Studies have reported that overexpression of IL-18 increased neutrophil infiltration and worsened kidney damage [53]. IL$1 \beta$ and IL-18 impair podocyte and endothelial function independent of inflammatory cell recruitment [54]. Under long-term hyperglycemia, the kidney's self-repairing ability is impaired, and continuous inflammation continues to aggravate kidney damage.

\section{Mitochondrial Antioxidants and mtDNA}

Mitochondria-targeted antioxidant mitoTEMPO has a significant effect on reducing the accumulation of 8 -oxoG in glomerular endothelial cells, reducing the level of 8-oxodG in urine, and has protective effects on endothelial cells and podocytes [12]. MitoQ protects against hyperglycemia-induced oxidative injury in tubular cells to maintain mitochondrial quality and also partially mediates mitophagy via Nrf2/PINK [55]. However, current clinical trials have shown that the efficacy of antioxidants (alpha lipoic acid, vitamin C, coenzyme Q10, mitoQ, etc.) on diabetic complications is not consistent [47, 56, 57]. Mitochondria inner membrane is highly impermeable, and this could decrease the efficacy of the supplements since they cannot cross the membrane and reach the targets [47].

In addition, in animal models of diabetes, Chinese herbal extracts such as Bombax ceiba L. leaves and isoorientin have also been shown to have a role in kidney protection, reducing mitochondrial oxidative stress, reducing ROS accumulation, and protecting mtDNA $[58,59]$. Salidroside and grape seed procyanidin B2 play a beneficial role against DKD in mice, which probably via AMPK/Sirt1/PGC- $1 \alpha$ mediated mitochondrial biogenesis. It also enhanced
mtDNA copy and electron transport chain proteins $[60,61]$. Chinese herbal extracts have achieved good results in the treatment of DKD, especially by improving mitochondrial oxidative stress. The safety and toxicity of traditional Chinese medicine still need to be verified with more rigorous experiments.

\section{Discussion}

DKD is a serious complication of diabetes. Studies have shown that early intensive glycemic control has certain benefits in delaying complications of diabetes [62]. In prediabetes, the epigenetics of mtDNA has begun to change. Before the occurrence of diabetic retinopathy, mtDNA methylation increased, and during the period of diabetic retinopathy, mtDNA methylation decreased [63]. This shows that mtDNA is very important in the occurrence and development of diabetes and its complications. Including mtDNA changes in the monitoring of diabetic patients is of great significance for long-term prognosis.

In the early stage of $\mathrm{DKD}$, the kidney showed a high filtration state; the ATP demand of kidney cells increased, causing mitochondrial oxidative stress and increased ROS production through downregulation of PGC- $1 \alpha$ and damage to mtDNA [45]. These mtDNA damages were first verified in glomerular endothelial cells, and secretion of 8-oxodG, a marker of mtDNA damage in urine, was increased [12]. Damaged endothelial cells reduce mitochondrial function, increase superoxide production, and damage mtDNA. Changes in the endothelial glycocalyx openings induce apoptosis of endothelial cells and cause impaired glomerular filtration. Eventually, albuminuria appears and renal function gradually decreases $[64,65]$. The early state of high glucose also induces the transfer of mtDNA into peripheral blood circulation. As a DAMP agent, ccf-mtDNA triggers a cascade of inflammation by activating inflammasomes (NLRP-3 and AIM2) and induces chronic sterile inflammation in the kidney (Figure 1). 
Although antioxidants can reduce oxidative stress in kidney tissue, they cannot completely reverse mtDNA changes, such as mtDNA deletion [30]. This suggests that oxidative stress is only one of the pathways for mtDNA damage. Exploring other damage pathways of mtDNA under high glucose conditions and using this as a target to intervene may become a new therapeutic approach for DKD.

Therefore, we believe that in the occurrence and development of $\mathrm{DKD}, \mathrm{mtDNA}$ changes are both the cause and the result. It is of great significance to incorporate the changes in mtDNA into the monitoring of DM patients: (1) the methylation level of mtDNA can be used as a predictive index for DM high-risk groups (obesity or family history of $\mathrm{DM})$; (2) the ccf-mtDNA copy number can be used as a predictor of DKD in the DM population with obesity or family history of DM; (3) ccf-mtDNA can also reflect chronic sterile inflammation in patients with DM or DKD; (4) mtDNA copy number of urine can be used as a diagnostic indicator of DKD and monitoring indicators of DKD progress; (5) ccf-mtDNA and urine mtDNA can be used as an indicator to improve mitochondrial oxidative stress by targeting mitochondrial antioxidants and Chinese herbal extracts; (6) exploring other damage pathways of mtDNA under high glucose conditions may become a new therapeutic approach for DKD.

Although in animal models and some small-scale clinical trials, it has been shown that changes in mtDNA are related to the progress of DKD $[16,23]$. At present, large-scale clinical trials are still needed to explore the threshold of mtDNA copy number for DKD diagnosis and the relationship between mtDNA and DKD prognosis. Besides, mtDNA copy number detection is expensive, which has become an important reason for limiting mtDNA as a routine monitoring of $\mathrm{DKD}$. Therefore, it is necessary to develop a simple and inexpensive detection method to determine the mtDNA copy number.

\section{Abbreviations}

\section{Glu: $\quad$ Glucose}

ROS: Reactive oxygen species

mtDNA: Mitochondrial DNA

mtETC: Mitochondrial electron transport chain

AMPK: Adenosine 5-monophosphate- (AMP-) activated protein kinase

Sirt1: $\quad$ Sirtuin 1

PGC-1 $\alpha$ : Peroxisome proliferator-activated receptor- $\gamma$ coactivator- $1 \alpha$

JNK: c-Jun N-terminal kinase

CaMKII: Calmodulin-dependent protein kinase II

Fis1: $\quad$ Mitochondrial fission protein 1

PKC: $\quad$ Protein kinase $\mathrm{C}$

eNOS: Endothelial nitric oxide synthase

VEGF: Vascular endothelial growth factor

TLR-4: Toll-like receptor-4

NF- $\kappa$ B: $\quad$ Nuclear factor kappa-B

TLR-6: Toll-like receptor-6

NLRP-3: NOD-like receptor protein-3
AIM2: $\quad$ Absent in melanoma 2

IL-1 $\beta$ : Interleukin- $1 \beta$

IL-18: Interleukin-18.

\section{Conflicts of Interest}

The authors declare that they have no conflicts of interest regarding the publication of this paper.

\section{Authors' Contributions}

YGW, YJH, and JWC were responsible for conception and design. YJH, YGW, and FXW performed data acquisition. YJH and YGW wrote the manuscript. YJH, YZ, YHC, and YGW carried out review and/or revision of the manuscript. All authors read and approved the final draft of the manuscript.

\section{Acknowledgments}

The authors gratefully thank Jingwei Chi for designing the review and Yue Zhou for valuable discussion.

\section{References}

[1] The Lancet Diabetes and Endocrinology, "Diabetic kidney disease: what does the next era hold?" The Lancet Diabetes \& Endocrinology, vol. 3, no. 9, p. 665, 2015.

[2] A. J. Collins, R. N. Foley, C. Herzog et al., "US renal data system 2010 annual data report," American Journal of Kidney Diseases, vol. 57, no. 1, pp. e1-526, 2011.

[3] J. M. Forbes and D. R. Thorburn, "Mitochondrial dysfunction in diabetic kidney disease," Nature Reviews Nephrology, vol. 14, no. 5, pp. 291-312, 2018.

[4] R. Z. Alicic, M. T. Rooney, and K. R. Tuttle, "Diabetic kidney disease: challenges, progress, and possibilities," Clinical Journal of the American Society of Nephrology, vol. 12, no. 12, pp. 2032-2045, 2017.

[5] Y. Hirakawa, T. Tanaka, and M. Nangaku, "Mechanisms of metabolic memory and renal hypoxia as a therapeutic target in diabetic kidney disease," Journal of Diabetes Investigation, vol. 8, no. 3, pp. 261-271, 2017.

[6] V. Tsimihodimos, T. D. Filippatos, and M. S. Elisaf, "SGLT2 inhibitors and the kidney: effects and mechanisms," Diabetes \& Metabolic Syndrome: Clinical Research \& Reviews, vol. 12, no. 6, pp. 1117-1123, 2018.

[7] K. Reidy, H. M. Kang, T. Hostetter, and K. Susztak, "Molecular mechanisms of diabetic kidney disease," Journal of Clinical Investigation, vol. 124, no. 6, pp. 2333-2340, 2014.

[8] A. Falkevall, A. Mehlem, I. Palombo et al., "Reducing VEGF-B signaling ameliorates renal lipotoxicity and protects against diabetic kidney disease," Cell Metabolism, vol. 25, no. 3, pp. 713-726, 2017.

[9] H. J. Sun, Z. Y. Wu, L. Cao et al., "Hydrogen sulfide: recent progression and perspectives for the treatment of diabetic nephropathy," Molecules, vol. 24, no. 15, p. 2857, 2019.

[10] Y.-C. Lin, Y.-H. Chang, S.-Y. Yang, K.-D. Wu, and T.-S. Chu, "Update of pathophysiology and management of diabetic kidney disease," Journal of the Formosan Medical Association, vol. 117, no. 8, pp. 662-675, 2018.

[11] J. C. Jha, C. Banal, B. S. M. Chow, M. E. Cooper, and K. Jandeleit-Dahm, "Diabetes and kidney disease: role of 
oxidative stress," Antioxidants \& Redox Signaling, vol. 25, no. 12, pp. 657-684, 2016.

[12] H. Qi, G. Casalena, S. Shi et al., "Glomerular endothelial mitochondrial dysfunction is essential and characteristic of diabetic kidney disease susceptibility," Diabetes, vol. 66, no. 3 , pp. 763-778, 2017.

[13] K. Sharma, B. Karl, A. V. Mathew et al., "Metabolomics reveals signature of mitochondrial dysfunction in diabetic kidney disease," Journal of the American Society of Nephrology, vol. 24, no. 11, pp. 1901-1912, 2013.

[14] L. D. Zheng, L. E. Linarelli, J. Brooke et al., "Mitochondrial epigenetic changes link to increased diabetes risk and earlystage prediabetes indicator," Oxidative Medicine and Cellular Longevity, vol. 2016, Article ID 5290638, 10 pages, 2016.

[15] A. Czajka, S. Ajaz, L. Gnudi et al., "Altered mitochondrial function, mitochondrial DNA and reduced metabolic flexibility in patients with diabetic nephropathy," EBioMedicine, vol. 2, no. 6, pp. 499-512, 2015.

[16] S. Akhtar and H. M. Siragy, "Pro-renin receptor suppresses mitochondrial biogenesis and function via AMPK/SIRT-1/ PGC- $1 \alpha$ pathway in diabetic kidney," PLoS One, vol. 14, no. 12, Article ID e0225728, 2019.

[17] S. Anderson, A. T. Bankier, B. G. Barrell et al., "Sequence and organization of the human mitochondrial genome," Nature, vol. 290, no. 5806, pp. 457-465, 1981.

[18] M. Tanaka and T. Ozawa, "Strand asymmetry in human mitochondrial DNA mutations," Genomics, vol. 22, no. 2, pp. 327-335, 1994.

[19] P. Sharma and H. Sampath, "Mitochondrial DNA integrity: role in health and disease," Cells, vol. 8, no. 2, p. 100, 2019.

[20] K. Ohkubo, A. Yamano, M. Nagashima et al., "Mitochondrial gene mutations in the tRNALeu (UUR) region and diabetes: prevalence and clinical phenotypes in Japan," Clinical Chemistry, vol. 47, no. 9, pp. 1641-1648, 2001.

[21] J. E. Carré and C. Affourtit, "Mitochondrial activity and skeletal muscle insulin resistance in kidney disease," International Journal of Molecular Sciences, vol. 20, no. 11, p. 2751, 2019.

[22] X. Deng, G. Yang, X. Zheng et al., "Plasma mtDNA copy numbers are associated with GSTK1 expression and inflammation in type 2 diabetes," Diabetic Medicine, 2019.

[23] G. Al-Kafaji, A. Aljadaan, A. Kamal, and M. Bakhiet, "Peripheral blood mitochondrial DNA copy number as a novel potential biomarker for diabetic nephropathy in type 2 diabetes patients," Experimental and Therapeutic Medicine, vol. 16, no. 2, pp. 1483-1492, 2018.

[24] H. Cao, J. Wu, J. Luo, X. Chen, J. Yang, and L. Fang, "Urinary mitochondrial DNA: a potential early biomarker of diabetic nephropathy," Diabetes/Metabolism Research and Reviews, vol. 35, no. 4, Article ID e3131, 2019.

[25] H. Jiang, X. Shao, S. Jia et al., "The mitochondria-targeted metabolic tubular injury in diabetic kidney disease," Cellular Physiology and Biochemistry, vol. 52, no. 2, pp. 156-171, 2019.

[26] H. Lu, V. Koshkin, E. M. Allister, A. V. Gyulkhandanyan, and M. B. Wheeler, "Molecular and metabolic evidence for mitochondrial defects associated with -cell dysfunction in a mouse model of type 2 diabetes," Diabetes, vol. 59, no. 2, pp. 448-459, 2010.

[27] S. Fujimaki and T. Kuwabara, "Diabetes-induced dysfunction of mitochondria and stem cells in skeletal muscle and the nervous system," International Journal of Molecular Sciences, vol. 18, no. 10, p. 2147, 2017.

[28] S. Tewari, J. M. Santos, and R. A. Kowluru, "Damaged mitochondrial DNA replication system and the development of diabetic retinopathy," Antioxidants \& Redox Signaling, vol. 17, no. 3, pp. 492-504, 2012.

[29] K. Chandrasekaran, M. Anjaneyulu, J. Choi et al., "Role of mitochondria in diabetic peripheral neuropathy: influencing the NAD+-dependent SIRT1-PGC-1 $\alpha$-TFAM pathway," International Review of Neurobiology, vol. 145, pp. 177-209, 2019.

[30] M. Kakimoto, T. Inoguchi, T. Sonta et al., "Accumulation of 8-hydroxy-2'-deoxyguanosine and mitochondrial DNA deletion in kidney of diabetic rats," Diabetes, vol. 51, no. 5, pp. 1588-1595, 2002.

[31] M. A. Nobrega, S. Fleming, R. J. Roman et al., "Initial characterization of a rat model of diabetic nephropathy," Diabetes, vol. 53, no. 3, pp. 735-742, 2004.

[32] L. V. Yuzefovych, V. M. Pastukh, M. V. Ruchko, J. D. Simmons, W. O. Richards, and L. I. Rachek, "Plasma mitochondrial DNA is elevated in obese type 2 diabetes mellitus patients and correlates positively with insulin resistance," PLoS One, vol. 14, no. 10, Article ID e0222278, 2019.

[33] P. Z. Wei, B. C.-H. Kwan, K. M. Chow et al., "Urinary mitochondrial DNA level is an indicator of intra-renal mitochondrial depletion and renal scarring in diabetic nephropathy," Nephrology Dialysis Transplantation, vol. 33, no. 5, pp. 784-788, 2018.

[34] C.-C. Chang, P.-F. Chiu, C.-L. Wu et al., "Urinary cell-free mitochondrial and nuclear deoxyribonucleic acid correlates with the prognosis of chronic kidney diseases," $\mathrm{BMC} \mathrm{Ne}$ phrology, vol. 20, no. 1, p. 391, 2019.

[35] P. Z. Wei and C. C. Szeto, "Mitochondrial dysfunction in diabetic kidney disease," Clinica Chimica Acta, vol. 496, pp. 108-116, 2019.

[36] J. Pedraza-Chaverri, L. G. Sánchez-Lozada, H. Osorio-Alonso, E. Tapia, and A. Scholze, "New pathogenic concepts and therapeutic approaches to oxidative stress in chronic kidney disease," Oxidative Medicine and Cellular Longevity, vol. 2016, Article ID 6043601, 21 pages, 2016.

[37] R. W. Taylor and D. M. Turnbull, "Mitochondrial DNA mutations in human disease," Nature Reviews Genetics, vol. 6, no. 5, pp. 389-402, 2005.

[38] Y. Zhang, J. Feng, Q. Wang et al., "Hyperglycaemia stressinduced renal injury is caused by extensive mitochondrial fragmentation, attenuated MKP1 signalling, and activated JNK-CaMKII-fis1 biological Axis," Cellular Physiology and Biochemistry, vol. 51, no. 4, pp. 1778-1798, 2018.

[39] X. Wang, L. Gao, H. Lin et al., "Mangiferin prevents diabetic nephropathy progression and protects podocyte function via autophagy in diabetic rat glomeruli," European Journal of Pharmacology, vol. 824, pp. 170-178, 2018.

[40] T. Honda, Y. Hirakawa, and M. Nangaku, "The role of oxidative stress and hypoxia in renal disease," Kidney Research and Clinical Practice, vol. 38, no. 4, pp. 414-426, 2019.

[41] P. Geraldes and G. L. King, "Activation of protein kinase C isoforms and its impact on diabetic complications," Circulation Research, vol. 106, no. 8, pp. 1319-1331, 2010.

[42] H. Noh and G. L. King, "The role of protein kinase C activation in diabetic nephropathy," Kidney International, vol. 72, no. 106, pp. S49-S53, 2007.

[43] Y.S. Kanwar, L. Sun, P. Xie, F.-Y. Liu, and S. Chen, "A glimpse of various pathogenetic mechanisms of diabetic nephropathy," Annual Review of Pathology: Mechanisms of Disease, vol. 6, no. 1, pp. 395-423, 2011.

[44] R. G. Amorim, G. D. S. Guedes, S. M. D. L. Vasconcelos, and J. C. D. F. Santos, "Kidney disease in diabetes mellitus: crosslinking between hyperglycemia, redox imbalance and 
inflammation," Arquivos brasileiros de cardiologia, vol. 112, no. 5, pp. 577-587, 2019.

[45] S. Yuan, X. Liu, X. Zhu et al., "The role of TLR4 on PGC- $1 \alpha-$ mediated oxidative stress in tubular cell in diabetic kidney disease," Oxidative Medicine and Cellular Longevity, vol. 2018, Article ID 6296802, 14 pages, 2018.

[46] M. Zhan, I. M. Usman, L. Sun, and Y. S. Kanwar, "Disruption of renal tubular mitochondrial quality control by myo-inositol oxygenase in diabetic kidney disease," Journal of the American Society of Nephrology, vol. 26, no. 6, pp. 1304-1321, 2015.

[47] J. M. Santos, G. Mohammad, Q. Zhong, and R. A. Kowluru, "Diabetic retinopathy, superoxide damage and antioxidants," Current Pharmaceutical Biotechnology, vol. 12, no. 3, pp. 352-361, 2011.

[48] Q. Zhang, M. Raoof, Y. Chen et al., "Circulating mitochondrial DAMPs cause inflammatory responses to injury," $\mathrm{Na}$ ture, vol. 464, no. 7285, pp. 104-107, 2010.

[49] C. A. Pereira, D. Carlos, N. S. Ferreira et al., "Mitochondrial DNA promotes NLRP3 inflammasome activation and contributes to endothelial dysfunction and inflammation in type 1 diabetes," Frontiers in Physiology, vol. 10, p. 1557, 2019.

[50] Z. Zhong, S. Liang, E. Sanchez-Lopez et al., "New mitochondrial DNA synthesis enables NLRP3 inflammasome activation," Nature, vol. 560, no. 7717, pp. 198-203, 2018.

[51] S. C. W. Tang and W. H. Yiu, "Innate immunity in diabetic kidney disease," Nature Reviews Nephrology, vol. 16, no. 4, pp. 206-222, 2020.

[52] J. H. Bae, S. I. Jo, S. J. Kim et al., "Circulating cell-free mtDNA contributes to AIM2 inflammasome-mediated chronic inflammation in patients with type 2 diabetes," Cells, vol. 8 , no. 4, p. 328, 2019.

[53] Y. Gonul, S. Kazandi, A. Kocak et al., "Interleukin-18 binding protein pretreatment attenuates kidney injury induced by hepatic ischemia reperfusion," The American Journal of the Medical Sciences, vol. 352, no. 2, pp. 200-207, 2016.

[54] K. Shahzad, F. Bock, W. Dong et al., "Nlrp3-inflammasome activation in non-myeloid-derived cells aggravates diabetic nephropathy," Kidney International, vol. 87, no. 1, pp. 74-84, 2015.

[55] L. Xiao, X. Xu, F. Zhang et al., "The mitochondria-targeted antioxidant MitoQ ameliorated tubular injury mediated by mitophagy in diabetic kidney disease via Nrf2/PINK1," Redox Biology, vol. 11, pp. 297-311, 2017.

[56] A. B. Oyenihi, A. O. Ayeleso, E. Mukwevho, and B. Masola, "Antioxidant strategies in the management of diabetic neuropathy," BioMed Research International, vol. 2015, Article ID 515042, 15 pages, 2015.

[57] O. Ilkun and S. Boudina, "Cardiac dysfunction and oxidative stress in the metabolic syndrome: an update on antioxidant therapies," Current Pharmaceutical Design, vol. 19, no. 27, pp. 4806-4817, 2013.

[58] G. Luan, Y. Wang, Z. Wang et al., "Flavonoid glycosides from fenugreek seeds regulate glycolipid metabolism by improving mitochondrial function in 3T3-L1 adipocytes in vitro," Journal of Agricultural and Food Chemistry, vol. 66, no. 12, pp. 3169-3178, 2018.

[59] G.-K. Xu, X.-Y. Qin, G.-K. Wang et al., "Antihyperglycemic, antihyperlipidemic and antioxidant effects of standard ethanol extract of Bombax ceiba leaves in high-fat-diet- and streptozotocin-induced type 2 diabetic rats," Chinese Journal of Natural Medicines, vol. 15, no. 3, pp. 168-177, 2017.

[60] H. Xue, P. Li, Y. Luo et al., "Salidroside stimulates the Sirt1/ PGC- $1 \alpha$ axis and ameliorates diabetic nephropathy in mice," Phytomedicine, vol. 54, pp. 240-247, 2019.
[61] L. Bao, X. Cai, Z. Zhang, and Y. Li, "Grape seed procyanidin B2 ameliorates mitochondrial dysfunction and inhibits apoptosis via the AMP-activated protein kinase-silent mating type information regulation 2 homologue 1-PPAR $\gamma$ co-activator- $1 \alpha$ axis in rat mesangial cells under high-dose glucosamine," British Journal of Nutrition, vol. 113, no. 1, pp. 35-44, 2015.

[62] I. H. de Boer, "Kidney disease and related findings in the diabetes control and complications trial/epidemiology of diabetes interventions and complications study," Diabetes Care, vol. 37, no. 1, pp. 24-30, 2014.

[63] A. J. Duraisamy, R. Radhakrishnan, B. Seyoum, G. W. Abrams, and R. A. Kowluru, "Epigenetic modifications in peripheral blood as potential noninvasive biomarker of diabetic retinopathy," Translational Vision Science \& Technology, vol. 8, no. 6, p. 43, 2019.

[64] A. Czajka and A. N. Malik, "Hyperglycemia induced damage to mitochondrial respiration in renal mesangial and tubular cells: implications for diabetic nephropathy," Redox Biology, vol. 10, pp. 100-107, 2016.

[65] B. J. Ballermann, "Contribution of the endothelium to the glomerular permselectivity barrier in health and disease," Nephron Physiology, vol. 106, no. 2, pp. p19-p25, 2007. 